\title{
Prediction of Shear Characteristics of Unsaturated Soil Based on BP Neural Network
}

\author{
Jia Lei LIANG ${ }^{1}$, Guang Ri JIN ${ }^{1, *}$, and Zhi Xie SHEN ${ }^{2}$ \\ ${ }^{1}$ Department of Civil Engineering, Yanbian University, Yanji, 133002, China \\ ${ }^{2}$ Department of Civil Engineering, Kyungpook National University, Daegu, South Korea
}

\begin{abstract}
The prediction model of shear strength parameters of unsaturated soil based on indoor test data is established by using BP neural network. Five kinds of network models with different number of hidden layer nodes are trained and studied, and the best network model is selected to conduct the prediction. The results show that the optimal BP network model is a single hidden layer structure of 8-16-2. Using this model to predict, the correlation coefficient and regression coefficient between the predicted value and the measured value are high, and the predicted result is reliable, so the method has certain practicability.
\end{abstract}

\section{Introduction}

Unsaturated soil exists widely in nature, and its strength is often involved in engineering. The research on unsaturated soil has always been the focus and difficulty in the field of geotechnical engineering. At present, the commonly used shear strength theories of unsaturated soil mainly include the effective stress shear strength theory proposed by Bishop [1] and the double stress state variable shear strength theory proposed by Fredlund [2]. The parameters in the shear strength expression are generally determined by the triaxial test method of unsaturated soil, which can control the drainage conditions and measure the pore water pressure. The accuracy of the test results is relatively high, so it is widely used in practical engineering, but the test procedure is tedious and takes a long time.

Artificial neural network has a strong ability of nonlinear mapping and adaptive learning, which has a wide range of applications in the prediction of nonlinear systems. Therefore, some scholars have tried to use the artificial neural network to study the characteristics of unsaturated soil. Miao Linchang et al. [3] established the relationship between the water content of unsaturated soil and the matric suction by using the artificial neural network. Xie Wenqiang et al. [4] predicted the undrained shear strength of cohesive soil by using the artificial neural network based on the field test data. Sheng Jinchang et al. [5] proposed to use artificial neural network method to predict the shear strength of rock joints. Therefore, in this paper, the shear strength parameters of unsaturated silty sand are predicted by establishing BP neural network model and using some simple and easy to measure physical indexes as influencing factors. This method not only saves time and labor, avoids tedious theoretical calculation, but also provides a method for the application of artificial neural network in unsaturated soil research.

\section{BP neural network}

Artificial neural network (ANN) is a network composed of a large number of processing units (neurons) which are widely interconnected. It is the abstraction, simplification and simulation of human brain, reflecting the basic characteristics of human brain. Among them, BP neural network (Back-Propagation Neural Network) is the most widely used, which is a multi-layer forward neural network. The activation function of the neuron is Sigmoid function,

$$
\sigma(s)=1 /\left(1+e^{-s}\right)
$$

the weight adjustment adopts the back propagation learning algorithm, that is, the error signal returns along the original connection channel. By modifying the weight and threshold of each layer of neuron, the error signal is minimized until the desired output is obtained. The typical BP neural network topology can be divided into three layers: input layer, hidden layer and output layer. It can realize any nonlinear mapping from input to output and has the ability of nonlinear function approximation. It is a system that simulates the information processing mechanism of the brain on the physical mechanism. It not only has the general computing ability to process the data, but also has the ability of learning and memory. The B.P network has been widely and successfully used in the pattern recognition, the image processing, the control optimization, the forecasting and the artificial intelligence.

\footnotetext{
* Corresponding author: grjin@ybu.edu.cn
} 


\section{The establishment and selection of model}

\subsection{Selection of training samples and prediction samples}

The sample data of the network model is the indoor test data, which is taken from the literature [6]. In the paper, through the axis-translation technique, the improved triaxial test system is used to control the matric suction of $0,5,20$ and $150 \mathrm{kPa}$ respectively, and the net confining pressure of 50, 100 and $200 \mathrm{KPa}$ respectively. The consolidated undrained test is carried out for the unsaturated soil with silt content of $0 \%, 5 \%, 10 \%$ and $20 \%$. In this study, 64 groups of data are collected in basic physical property test and triaxial shear test, including 48 groups of learning data and 16 groups of prediction data.

\subsection{Establishment of BP network model}

\subsubsection{Analysis of factors influencing the shear strength of unsaturated soil}

(1) Matrix suction: The shear strength increases with the increase of matrix suction; for expansive soil, the suction increases the effective stress between particles and increases the dilatancy potential of soil.

(2) Initial void ratio: For the same kind of soil, the smaller the initial void ratio is, the tighter the soil is, and the higher the shear strength of the intrinsic structure is.

(3) Void ratio: It affects the shape of soil water characteristic curve, and then affects the shear strength of unsaturated soil.

(4) Water content: In the range of low water content, the shear strength of unsaturated soil decreases with the increase of water content; with the increase of water content, the cohesion of soil increases first and then decreases. For unsaturated silty clay, the internal friction angle decreases with it, but it has little effect on the internal friction angle of loess.

(5) Dry density: When the saturation is the same, the internal friction angle and cohesion of unsaturated silty clay increase with the increase of dry density.

(6) Clay content: The higher the clay content, the greater the cohesion, the smaller the internal friction angle, and the lower the shear strength when the water content is the same.

It can be seen from this that there are many factors that affect the shear strength of unsaturated soil, and the influence degree of various factors is different. Therefore, the prediction of the shear strength of unsaturated soil is relatively complex. It is necessary to use artificial neural network to effectively combine the advantages of various factors to provide more accurate prediction.

\subsubsection{Model structure design}

BP neural network requires that all neurons between adjacent layers to be fully connected, but all input neurons are independent of each other and cannot have direct linear connection. According to the test data and influence factor analysis, silt content, void ratio, maximum void ratio, minimum void ratio, D10, D30, D60 and matrix suction are selected as input factors, 8 items in total, the cohesion coefficient and internal friction angle are selected as output factors. The input and output information of the data used for learning is summarized as Table 1. Among them, all the data of 0 is approximately converted to 0.0001 , which can reduce the difficulty of neural network training and the number of training.

Table. 1. Input and output information of unsaturated soil shear characteristics prediction model

\begin{tabular}{|c|c|c|}
\hline Input information & Minimum value & Maximum value \\
\hline Silt content(\%) & 0.0001 & 30 \\
\hline Void ratio & 0.744 & 1.048 \\
\hline Maximum void ratio & 1.181 & 1.295 \\
\hline Minimum void ratio & 0.606 & 0.849 \\
\hline $\mathrm{D} 10(\mathrm{~mm})$ & 0.01 & 0.19 \\
\hline $\mathrm{D} 30(\mathrm{~mm})$ & 0.12 & 0.22 \\
\hline $\mathrm{D} 60(\mathrm{~mm})$ & 0.23 & 0.25 \\
\hline Matric suction(kPa) & 0.0001 & 150 \\
\hline Output information & Minimum value & Maximum value \\
\hline $\mathrm{c}$ & 0.0001 & 20 \\
\hline$\phi$ & 33.6 & 35.3 \\
\hline
\end{tabular}

At present, there is no theoretical guidance for the selection of the number of the hidden layer nodes. Increasing the number of layers can further reduce the error and improve the accuracy, but complicate the network at the same time, thus increasing the training time of the network weight. Referring to the research results of Xia Kewen et al. [7], this time, the number of the hidden layer nodes is set between one to three times of the input layer, a total of five kinds.

The network algorithm adopts the additional momentum method. This algorithm by adding a momentum factor, records the influence of the last connection weight coefficient change. Therefore, the adjustment of the connection weight coefficient will contain a part of the adjustment of the last connection weight coefficient, which can reduce the oscillation trend in the learning process and improve the convergence of the network.

The connection strength is 16 kinds of combinations in the range of 0.01-0.5 and 0.1-0.7. The initial learning efficiency is 0.1 and the momentum factor is 0.9 , the maximum training times is 400000 , and the allowable error value is 0.0001 . That is to say, when the difference between the network output and the sample is less than 0.0001 , the correction of the network weight is stopped. The structure information of the learning model is shown in Table 2. 
Table. 2. The structure of unsaturated soil shear characteristics learning model

\begin{tabular}{|c|c|c|}
\hline Neural network structure & Connection strength & Learning times \\
\hline $8-8-2$ & \multirow{5}{*}{$\begin{array}{cl}0.01 / 0.1, & 0.01 / 0.3 \\
0.01 / 0.5, & 0.01 / 0.7 \\
0.1 / 0.1, & 0.1 / 0.3 \\
0.1 / 0.5, & 0.1 / 0.7 \\
0.3 / 0.1, & 0.3 / 0.3 \\
0.3 / 0.5, & 0.3 / 0.7 \\
0.5 / 0.1, & 0.5 / 0.3 \\
0.5 / 0.5, & 0.5 / 0.7\end{array}$} & \multirow{5}{*}{400,000} \\
\hline $8-12-2$ & & \\
\hline $8-16-2$ & & \\
\hline $8-20-2$ & & \\
\hline $8-24-2$ & & \\
\hline
\end{tabular}

\subsection{Selection of the best artificial neural network model}

In order to compare the learning and prediction effect in neural network, according to the evaluation principle and practice, MAE (mean absolute error), MAPE (mean absolute percentage error), MSE (mean square error) and other error evaluation indexes are usually used to carry out a comprehensive measurement and evaluation. In this paper, RMSE (root mean square error) is selected to evaluate the network performance.

$$
R M S E=\sqrt{ }\left[\sum e_{n}^{2} /(n-1)\right]
$$

The $e_{n}$ is the deviation between the predicted and the actual value for the number $\mathrm{n}, \mathrm{n}$ is the number of samples. RMSE is used to measure the deviation between the observed value and the real value, which can well reflect the measurement accuracy, and especially is very sensitive to the large or small errors in a group of measurements. Through self-learning of neural network, collate the data in each learning result file. RMSE results of BP network model under different number of hidden layers and connection strength are shown in Figure 1 and Figure 2.

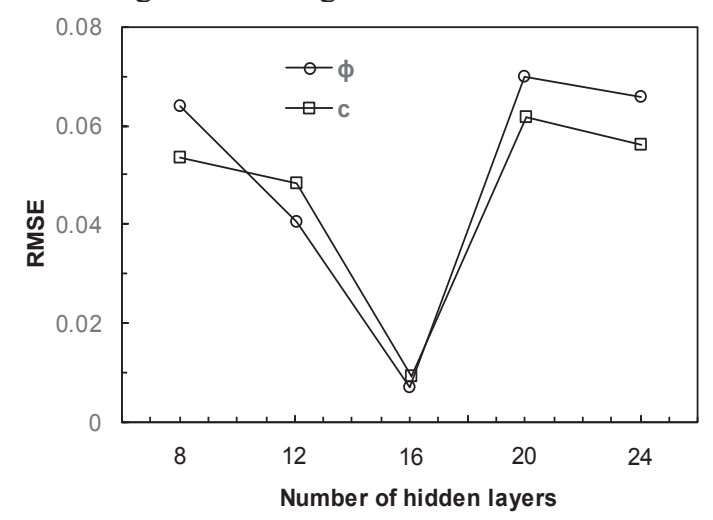

Fig. 1. RMSE varies with the number of hidden layers

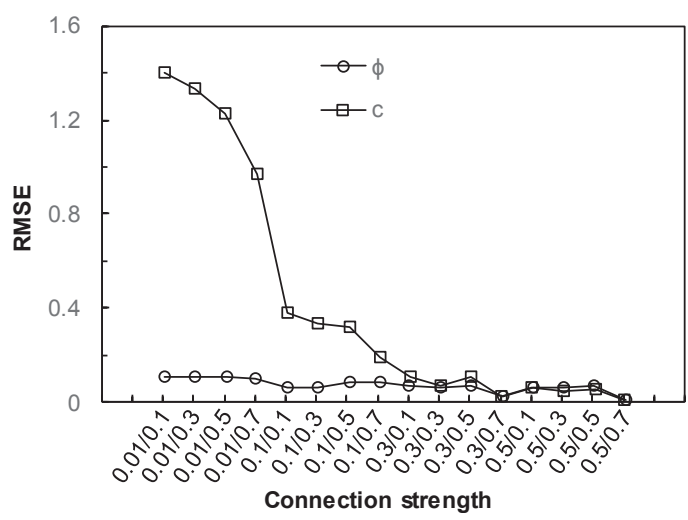

Fig. 2. RMSE varies with the Connection strength

There are two output factors of BP network model. When selecting the best artificial neural network, it is unreasonable to find the best composition according to each parameter. Therefore, the structure with the minimum sum of RMSE values of the two parameters is selected. According to Figure 1 and Figure 2, when there are eight input layers and two output layers, the optimal number of hidden layer nodes is 16, the optimal connection strength is $0.5 / 0.7$, and the RMSE $(\mathrm{c} / \phi)$ is $0.00934 / 0.00696$, and the correlation coefficient of learning is 0.99 , which proves that there is a strong correlation between the influencing factor and the output result and this method is feasible. The structure information of the optimal neural network model is shown in table 3.

Table. 3. The structure information of the optimal neural network model

\begin{tabular}{|c|c|}
\hline Input layers number & 8 \\
\hline Hidden layer nodes number & 16 \\
\hline Output layers number & 2 \\
\hline Learning rate & 0.5 \\
\hline Motivation & 0.7 \\
\hline Correlation coefficient & 0.99 \\
\hline RMSE $(c / \phi)$ & $0.00934 / 0.00696$ \\
\hline
\end{tabular}

\section{Analysis of prediction results}

The best neural network model is used to predict 16 groups of data. The predicted value is the abscissa and the measured value is the ordinate. as shown in Figure 3. It can be seen in the figure that the data points are distributed on both sides of the ideal regression curve $(\mathrm{X}=\mathrm{Y})$. Meanwhile, according to the existing data points, regression fitting is carried out for the predicted value and the measured value, it is a linear regression equation. The regression coefficients of $\mathrm{c}$ and $\phi$ are 0.99 and 0.96 respectively, indicating that the measured value and the predicted value have a high degree of fitting, and the correlation coefficients are 0.99 and 0.98 respectively, which proves that the correlation between the two is high, indicating that the prediction data is reliable. 


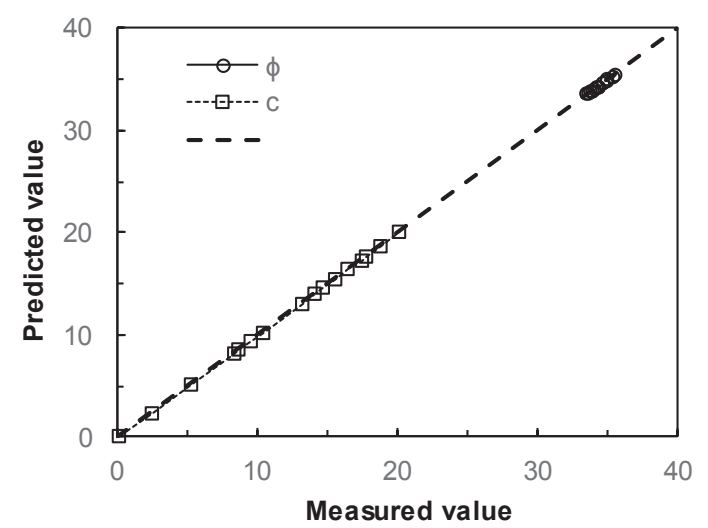

Fig. 2. Comparison between measured value and predicted value

Generally, 3 4 samples should be prepared for triaxial test, which are respectively applied with different surrounding pressure and consolidated shear, recorded and calculated to obtain the shear strength parameters of soil samples, the whole process takes a long time and the preparation and installation process is not suitable for operation. However, after using artificial neural network to establish the prediction model, the corresponding input data are measured through simple test, and the corresponding shear strength parameters are predicted directly by using the model, which takes much less time than the traditional method, so this method has a wide prospect in practical engineering application.

\section{Conclusions}

1. The above results show that the BP neural network prediction model can successfully predict the shear strength parameters of unsaturated soil by directly modeling using data, and the fitting degree and correlation between the prediction results and the measured values are high, which provides a method for scientific prediction of the shear characteristics of unsaturated soil.

2. By changing the number of hidden layer nodes, an optimized BP network model is established, and the optimal model structure is obtained through comparative analysis: the number of input layers is eight, the number of hidden layers is 16 , the number of output layers is two, and the connection strength is $0.5 / 0.7$.

3 . Through the analysis of the results, the selection of the influencing factors of the BP network model has a certain practicality. For different research ideas and influencing factors, it still needs to be further explored.

4. Based on the simple and easily measured basic physical property test data in the laboratory, combined with the computer and neural network technology, through continuous improvement, the strength characteristics of soil can be evaluated more quickly, which is conducive to better application in practical engineering in the future.

\section{References}

1. A.W. BISHOP. Tek. Ukebl, 106,113-143(1959)

2. D.G. FREDLUND, N.R. MORGENSTEM, R.A. WIDGER. Can. Geotech. J, 15, 313-321(1978)

3. L.C. Miao, M.L. Yan, Z.Z. Yin.J. J. Hohai Univ., Nat. Sci. 27,66-69(2012)

4. W.Q. Xie, G.J. Cai, R. Wang, J.M. Zhang. Chin Civil Eng J, 52,35-41(2019)

5. J.CH. Sheng, B.Y. Su. J. Hohai Univ., Nat. Sci. 03,107-110(2001)

6. ZH.X. Shen. The Analysis and Prediction of Unsaturated Characteristics for Nak-dong River Silty Sand.(Central Library of Kyungpook National University, 2011)

7. K.W. Xia, CH.B. Li, J.Y. Shen. Computer Science, 32,143-145(2005) 